\title{
Abbreviations and Editions
}

\author{
Abbreviations \\ BNJ: \\ I. Worthington (ed.), Brill's New Jacoby (http://www.brillonline.nl), 2007-. \\ CPF: \\ Corpus dei Papiri Filosofici Greci e Latini, Firenze, 1982-. \\ CPF $\quad=$ Corpus dei Papiri Filosofici Greci e Latini. Testi e Lessico nei papiri di cultura \\ $1.1^{\star \star \star}$

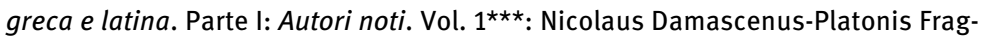 \\ menta, Firenze, 1999. \\ EGEF: $\quad$ C. Tsagalis (ed.), Early Greek Epic Fragments. I, Antiquarian and Genealogical Po- \\ etry, Berlin/Boston 2017. \\ EGM: $\quad$ R.L. Fowler (ed.), Early Greek Mythography. I, Text and Introduction, Oxford 2000; \\ II, Commentary, Oxford 2013.
}

FGrHist: $\quad$ F. Jacoby (ed.), Die Fragmente der griechischen Historiker. Erster Teil: Genealogie und Mythographie (Leipzig 1923, Leiden 1957²); Zweiter Teil: Zeitgeschichte (1926-1930, 1961-1963²); Dritter Teil: Geschichte von Städten und Völkern (Horographie und Ethnographie) $\left(1940,1955-1957^{2}\right)$.

FHG: $\quad$ C. Müller (ed.), Fragmenta Historicorum Graecorum. I, with T. Müller, Paris 1841; II, 1848; IV 1851; V 1, 1873; V 2 ed. V. Langlois, 1872.

FHS\&G: $\quad$ W. Fortenbaugh/P. Huby/R. Sharpied/D. Gutas (eds.), Theophrastus of Eresus, Sources for his Life, Writings, Thought and Influence, 2 vols., Leiden 2016.

IACP: $\quad$ M.H. Hansen/T.H. Nielsen (eds.), An Inventory of Archaic and Classical Poleis, Oxford 2004.

IG: Inscriptiones Graecae, Berlin 1873-.

LGPN: $\quad$ Lexicon of Greek Personal Names, http://www.lgpn.ox.ac.uk.

LIMC: $\quad$ Lexicon iconographicum mythologiae classicae, Zürich 1981-1999.

OF: $\quad$ A. Bernabé (ed.), Poetae Epici Graeci. Testimonia et Fragmenta. Pars II, fasc. 1-2: Orphicorum et Orphicis similium testimonia et fragmenta (Leipzig 2004-2005); Pars II, fasc. 3: Musaeus-Linus-Epimenides-Papyrus Derveni-Indices (Leipzig 2007).

PEG: $\quad$ A. Bernabé (ed.), Poetae Epicae Graeci. Pars I: Testimonia et fragmenta, Leipzig 1987.

PMG: $\quad$ M. Davies (ed.), Poetae Melici Graeci, Oxford 1962.

PMGF: $\quad$ M. Davies (ed.), Poetarum Melicorum Graecorum Fragmenta, I, Oxford 1991.

RE: A. Pauly/G. Wissowa/W. Knoll (eds.), Paulys Real-Encyclopädie der classischen Altertumswissenschaft, Stuttgart 1893-1972.

SEG: $\quad$ Supplementum Epigraphicum Graecum, Leiden 1923-.

SH: $\quad$ H. Lloyd-Jones/P.J. Parsons (eds.), Supplementum Hellenisticum, Berlin 1983.

TrGF: $\quad$ Tragicorum Graecorum Fragmenta. I, Didascaliae tragicae, Catalogi tragicorum et tragoediarum, Testimonia et fragmenta tragicorum minorum, eds. B. Snell, R. Kannicht (Göttingen, 1986); II, Fragmenta adespota, eds. B. Snell, R. Kannicht (1981); III, Aeschylus, ed. S. Radt (1985); IV, Sophocles, ed. S. Radt (1977); V, Euripides (2 vols.), ed. R. Kannicht (2004). 
- For ancient Greek authors, abbreviations are those found in the Oxford Classical Dictionary, with a few trivial divergences.

- For ancient Latin authors, the abbreviations in the Thesaurus Linguae Latinae have been followed.

- Abbreviations of periodicals are those recommended in the American Journal of Philology (AJA), 95 (1991), 1-16 (and available at https://www.ajaonline.org/submissions/abbreviations).

- The text of Acusilaus' fragments and the apparatus are based on Fowler's EGM, with modifications and updates. I have also used Fowler's numeration, which is almost identical with Jacoby's.

- Fragments where Acusilaus' name has been restored and supplemented are marked with *. When he is not mentioned, but the content of the text testifies to an ascription to him, fragments are marked with **.

\section{Editions}

Modern editions of texts are those listed in EGM, with the exception of:

- M. Broggiato (ed.), Cratete di Mallo. I frammenti: edizione, introduzione e note, La Spezia 2001.

- F. Pontani (ed.), Scholia Graeca in Odysseam, I-, Roma 2010-.

- S. Radt (ed.), Strabons Geographika, Göttingen 2002-2011.

- H. van Thiel (ed.), D-Scholia to the lliad, available on line at http://kups.ub.uni-koeln.de/1810/.

The text of the fragmentary authors is cited from the following editions:

Aristotle and his V. Rose (ed.), Aristotelis qui ferebantur librorum fragmenta, Leipzig $1886^{3}$. school:

F. Wehrli (ed.), Die Schule des Aristoteles, Basel/Stuttgart 1967-1969².

Early Mythogra- EGM. For authors not included in EGM, quotations are from FGrHist.

phers/

Historians:

Demetrius of

R. Gaede (ed.), Demetrii Scepsii quae supersunt, Diss. Greifswald 1880.

Scepsis:

Mnaseas of

P. Cappelletto (ed.), I frammenti di Mnasea. Introduzione, testo e commento,

Patara:

Presocratics: Milano 2003.

A. Laks/G.W. Most (eds.), Early Greek Philosophy, Cambridge MA/London 2016.

H. Diels (ed.), Die Fragmente der Vorsokratiker, Berlin 1951-1952 ${ }^{6}$

(ed. W. Kranz).

Pherecydes of H.S. Schibli (ed.), Pherekydes of Syros, Oxford 1990.

Syros:

Comic Poets: $\quad$ R. Kassel/C. Austin (eds.), Poetae Comici Graeci, Berlin/New York, 1983-1998.

Epic Poets: $\quad$ PEG, EGEF (see above).

M.L. West (ed.), Greek Epic Fragments, Cambridge MA/London 2003. 
Hesiod:

lambic and

Elegiac Poets:

Lesbian Poets:

Lyric Poets:

Bacchylides:

Callimachus:

Nicander of

Colophon:

Pindar:

Simonides:

Stesichorus:

Tragic Poets:
R. Merkelbach/M.L. West (eds.), Fragmenta Hesiodea, Oxford 1967.

G.W. Most (ed.), Hesiod I: Theogony. Works and Days. Testimonia (Cambridge MA/London 2006); II: The Shield. Catalogue of Women. Other fragments (Cambridge MA/London 2007).

M.L. West (ed.), Iambi et Elegi Graeci ante Alexandrum cantati, I-II, Oxford, 1989-1992.

E.M. Voigt (ed.), Sappho et Alcaeus, Amsterdam 1971.

PMG/PMGF (see above).

H. Maehler (ed.), Bacchylidis carmina cum fragmentis, Leipzig $2003^{11}$.

R. Pfeiffer (ed.), Callimachus I: Fragmenta, Oxford 1949.

G. Massimilla (ed.), Callimaco. Aitia, Libri primo e secondo, Pisa 1996; Libri terzo e quarto, Pisa 2010.

A. Harder (ed.), Callimachus: Aetia (2 vols.), Oxford/New York 2014.

O. Schneider (ed.), Nicandrea, Leipzig 1856.

A.S.F. Gow/A.F. Scholfield (eds.), Nicander. Poems and Poetical Fragments, Cambridge 2014.

B. Snell/H. Maehler (eds.), Pindari carmina cum fragmentis, Leipzig 1975.

I. Rutherford (ed.), Pindar's Paeans. A Reading of the Fragments with a Survey of the Genre, Oxford 2001.

O. Poltera (ed.), Simonides lyricus. Testimonia und Fragmente. Einleitung, kritische Ausgabe, Übersetzung und Kommentar, Basel 2008.

M. Davies/P. Finglass (eds.), Stesichorus, The Poems, Cambridge 2014.

TrGF (see above).

\section{Works Cited in the Apparatus by Author's Name}

Aegius

Barnes

Bergk

Blomfield

Böckh

Boll

Broggiato

Bücheler
B. Aegius (ed.), Apollodori Atheniensis Bibliotheces sive de deorum origine, Roma 1555.

J. Barnes (ed.), Homeri Odyssea et in eandem scholia sive interpretatio veterum, Cambridge 1711.

Th. Bergk, “Lesefrüchte: Zu den Pindarischen Scholien”, in: Neue Jahrbücher für Philologie und Paedagogik 135, 1878, 37-46.

J. Blomfield, “Sapphonis Fragmenta", in: Museum Criticum 1, 1826, 1-31.

A. Böckh (ed.), Pindari opera quae supersunt. II 1, Scholia, Leipzig 1819. apud Deubner

M. Broggiato (ed.), Cratete di Mallo, I frammenti: edizione, introduzione e note, La Spezia 2001.

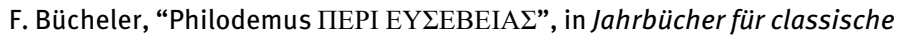
Philologie 11, 1865, 513-541. 


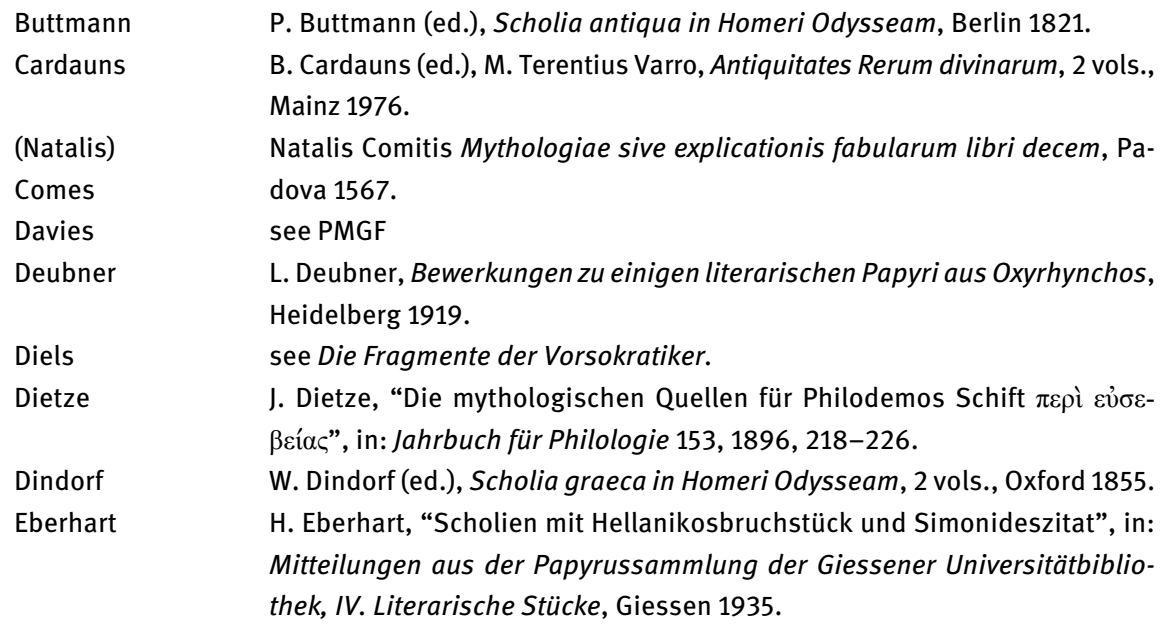

Facius

Gomperz

Gomperz $^{2}$

Gronovius

Hercher

Heyne

Heyne $^{2}$

Holwerda

Hommel

Jacoby

Janko

Jones

Kassel

Kaye

Keil

Kirchhoff

Körte

Kordt

Kramer

Lasserre

Lobeck
J.F. Facius (ed.), Pausaniae Graeciae Descriptio, Leipzig 1794-1796.

T. Gomperz, "Zu Philodem $\pi \varepsilon \rho \grave{~ \varepsilon u ̉ \sigma \varepsilon ß \varepsilon i ́ a c ", ~ i n: ~ Z e i t s c h r i f t ~ f u ̈ r ~ d i e ~}$ österreichischen Gymnasien, 15, 1864, 637-648, 731-736.

Herkulanische Studien. II, Philodem, Über Frömmigkeit, Leipzig 1866.

J. Gronovius, Thesaurus Graecorum Antiquitatum 1, Lyon 1667.

R. Hercher (ed.), Apollodori Bibliotheca, Berlin 1874.

C.G. Heyne (ed.), Ad Apollodori Atheniensis bibliothecam notae cum commentatione de Apollodoro ... et cum Apollodori fragmentis, Göttingen 1783. In: Académie des inscriptions et belles-lettres: Mémoires 1 (1815), 377.

D. Holwerda (ed.), Prolegomena de comoedia, scholia in Acharnenses, Equites, Nubes, pars I, Groningen 1977.

A. Hommel (ed.), Platonis Convivium, Leipzig 1834.

see FGrHist

apud EGM

H.L. Jones (ed.), Strabo, Geography, Books 10-12, Harvard 1928. apud EGM

J. Kaye, “On Certain Greek Historians mentioned by Dionysius of Halicarnassus", in: Museum Criticum 1, 1814, 216-225.

H. Keil (ed.), Apollonii Argonautica. II, Prolegomena et scholia vetera, Leipzig 1854.

A. von Kirchhoff, Die homerische Odyssee, Berlin $1879^{2}$. apud Kordt

A. Kordt, De Acusilao, Diss. Basel 1903.

G. Kramer (ed.), Strabonis Geographica, 3 vols., Berlin 1844-1852.

F. Lasserre (ed.), Strabon, Géographie. Tome VII. Livre X, Paris 1971.

C.A. Lobeck, Aglaophamus. De theologiae mysticae Graecorum causis, 2 vols., Könisberg 1829. 


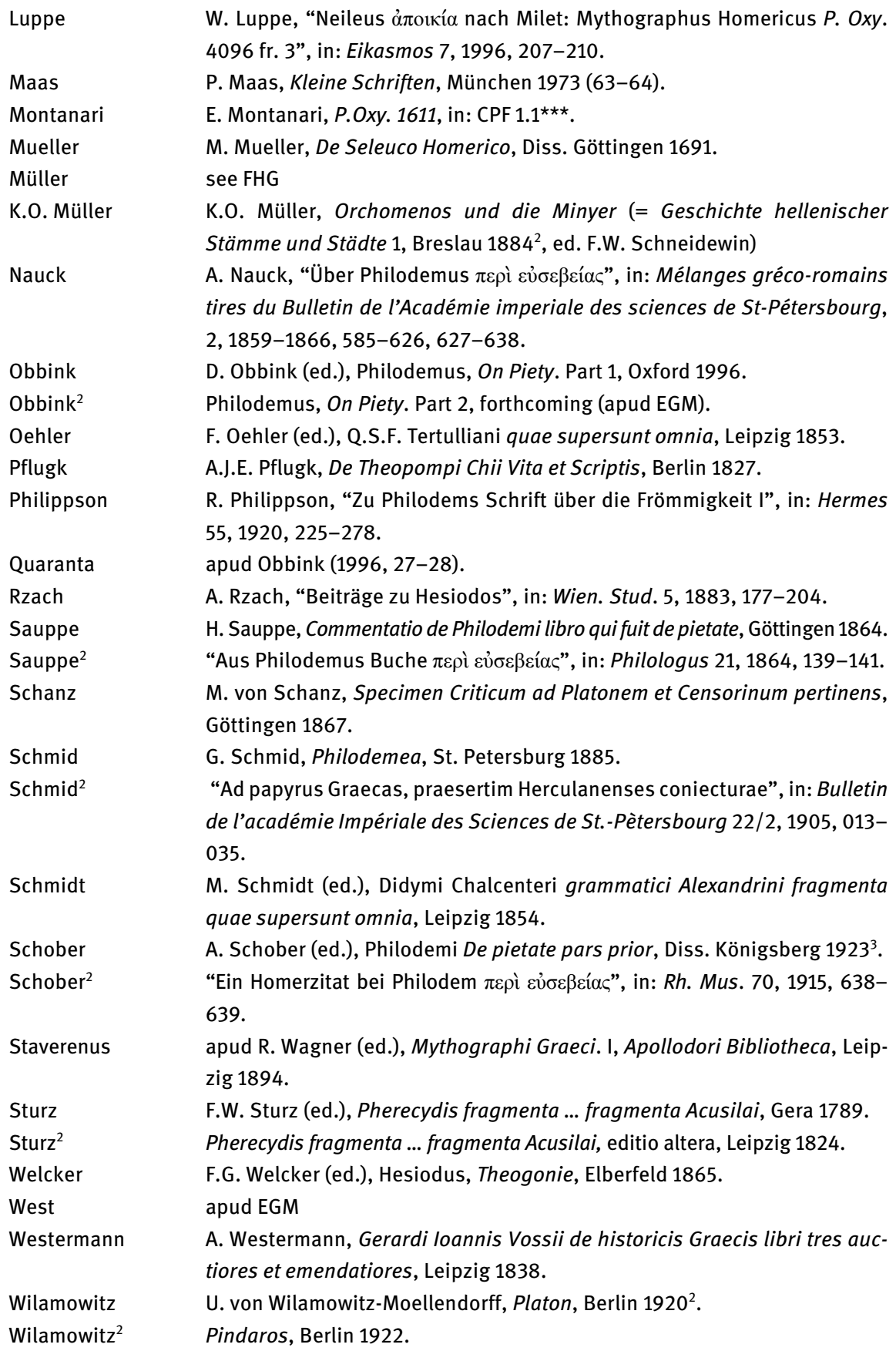


Wilamowitz ${ }^{3}$

"Pherekydes", in: SPAW 1926, 125-146 (= Kleine Schriften, 5.2, Berlin 1937, 127-156). 